\title{
Drainage of left hepatic vein into coronary sinus associated with other systemic venous anomalies
}

\author{
Ronald L. van der Horst, William S. Winship, and Mervyn S. Gotsman \\ From the Cardio-Thoracic Unit, Wentworth Hospital, and the University of Natal, Durban, \\ South Africa
}

This report describes a case with double hepatic venous drainage into the right atrium. The right hepatic veins, the inferior cava, and the pulmonary veins drained normally. The left hepatic veins drained into the coronary sinus and via the coronary ostium into the right atrium. A persistent left superior vena cava, draining the left upper half of the body into the coronary sinus and right atrium, was also present, and the right superior vena cava drained the right upper half of the body.

Associated defects were a sinus venosus atrial septal defect and valvular pulmonary stenosis.

The purpose of this report is to describe the abnormalities found in an infant who had abnormal drainage of the left hepatic vein into the coronary sinus. The diagnosis was established during life by cardiac catheterization and angiocardiography, and the associated lesions included a sinus venosus atrial septal defect, valvular pulmonary stenosis, and persistence of the left superior vena cava draining into the coronary sinus and right atrium. The right hepatic veins and the pulmonary veins drained normally. This rare malformation is not mentioned in 8 major texts on cardiac malformations (Nadas, 1963; Gasul, Arcilla, and Lev, 1966; Keith, Rowe, and Vlad, 1967; Wood, I968; Kjellberg et al., I959; Taussig, 1960; Moss and Adams, 1968; Edwards et al., 1965; Watson, 1968).

\section{Case report}

An African male infant, aged 2 years, presented with a history of frequent respiratory tract infections and failure to thrive. On clinical examination, he had an ejection systolic murmur in the pulmonary area, with wide, fixed splitting of the second heart sound. The electrocardiogram showed a mean frontal QRS axis of $-120^{\circ}$ and right ventricular hypertrophy with an $\operatorname{RsR}^{1}$ pattern in the right praecordial leads. The chest $x$-ray is shown in Fig. I. The widened superior mediastinum and pulmonary plethora suggested anomalous pulmonary venous drainage of the supracardiac type. At diagnostic cardiac catheterization, a peak systolic gradient of $40 \mathrm{~mm}$. $\mathrm{Hg}$ was present across the pulmonary valve and a left-to-right shunt of $\mathrm{I} I \mathrm{l} . / \mathrm{min} . / \mathrm{m}^{2}$. at atrial level was detected. The pulmonary to systemic blood flow ratio was
2.5:1. Cine-angiocardiography, injecting selectively into the right ventricle, left atrium and ventricle, aorta, pulmonary veins, and coronary sinus, showed the following abnormal anatomical features: a thickened pulmonary valve due to pulmonary stenosis; a sinus venosus atrial septal defect; normal drainage of all the pulmonary veins into the left atrium; and a normal left ventricle and aorta. The innominate vein was hypoplastic; the right superior vena cava drained the right subclavian and jugular veins and then

FIG. I Chest $\mathrm{x}$-ray showing a widened superior mediastinum due to the persistent left superior vena cava and thymic shadow, and a slight increase in pulmonary vascularity in keeping with the left-to-right shunt.

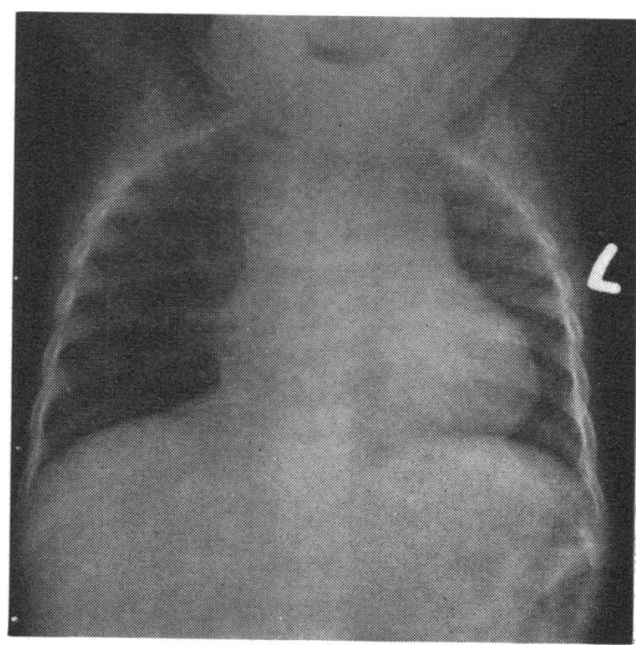


entered the right atrium. The left superior vena cava drained the left subclavian and jugular veins and entered the right atrium via the coronary sinus (Fig. 2). The right hepatic veins joined the inferior vena cava and entered the right atrium normally, but the left hepatic veins drained into the coronary sinus and thence into the right atrium (Fig. 3).

\section{Discussion}

This abnormality of hepatic vein drainage into the coronary sinus has been reported for several reasons. It is a rare condition, scantily described in the published reports. It should be shown before cardiac operation is undertaken using the heart-lung machine, because it may not be possible to control the venous return if it is present. Moreover, with present advances in hepatic surgery and transplantation, the anatomy of the hepatic venous drainage has assumed greater importance.

Left axis deviation in patients with secundum atrial septal defects has been reported by Harrison and Morrow (1963), Hastreiter and Rodriguez-Coronel (1968), and others. In our patient the mean QRS axis is directed superiorly and to the left. Angiographically a high sinus venosus defect is clearly seen with normal drainage of all the pulmonary veins. The frequent association between a sinus venosus atrial septal defect and persistence

FIG. 2 A selected frame from the cineangiogram showing bilateral superior vena cavae and the hypoplastic innominate vein. Contrast medium extravasated into the superior + mediastinum (upper arrow). The left hepatic vein is also shown (lower arrow).

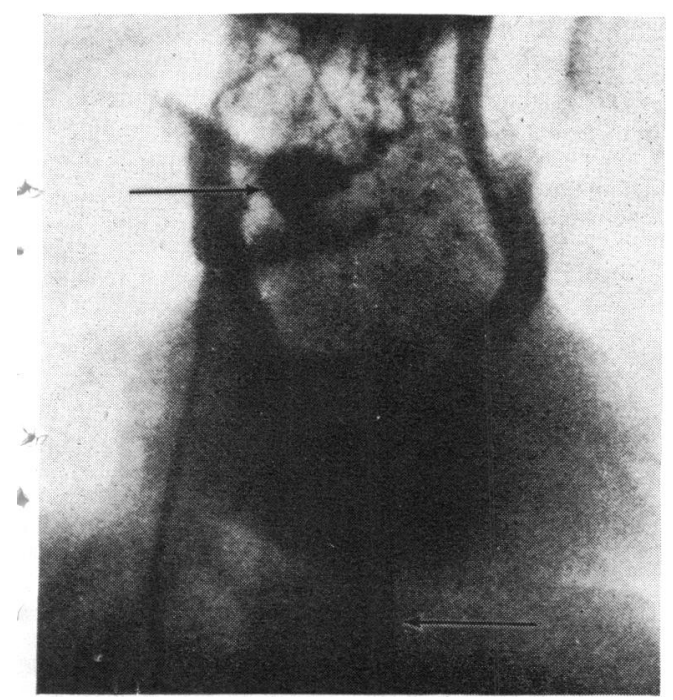

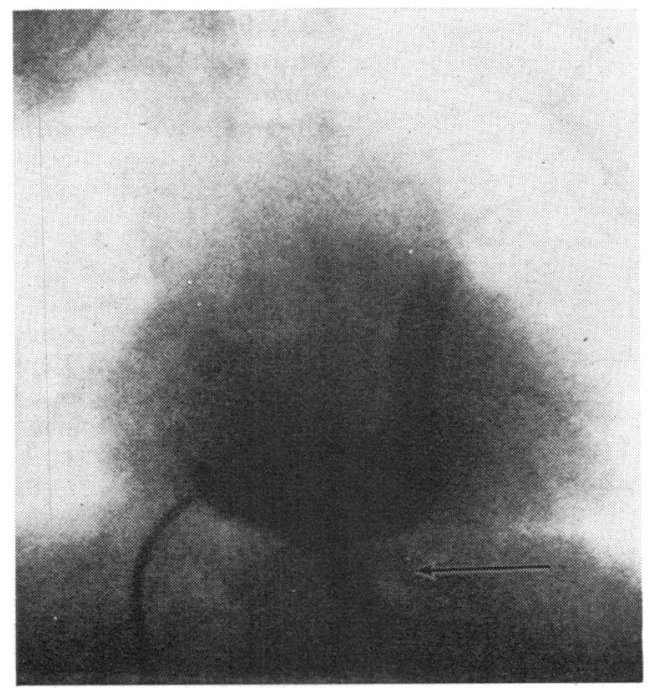

FIG. 3 Selective angiogram into the coronary sinus, showing the left hepatic vein and superior vena cava draining into the coronary sinus and right atrium.

of the left superior vena cava has been commented upon by Fleming and Gibson (1964).

Embryological considerations The embryological significance of finding veins from the left lobe of the liver which drain into the coronary sinus, contributes towards an understanding of the development of the normal systemic venous system.

Persistence of the left superior vena cava (persistence of the left anterior cardinal vein), which then drains into the left duct of Cuvier (the left sinus horn) and thence into the venous sinus (sinus venosus) is not uncommon. The venous sinus end becomes the coronary ostium which drains into the right atrium. If the left superior vena cava remains patent at both ends, as in our case, it drains the left cranial and arm veins to the right atrium, so that the innominate vein is either small or undeveloped and the right superior vena cava relatively smaller than normal.

The early development of the heart before the $4 \mathrm{~mm}$. stage includes the formation of the primitive heart tube, originally a bilateral symmetrical anlage in the cardiogenic area. The developing liver and systemic veins below the heart are also centrally situated and bilaterally represented. The veins represented 1-5, appropriately labelled, in Fig. 4, open into the venous sinus, but as this chamber tapers (bends) laterally to the right in situs solitus, to form the right atrium, the developing liver also increases in size on the right 


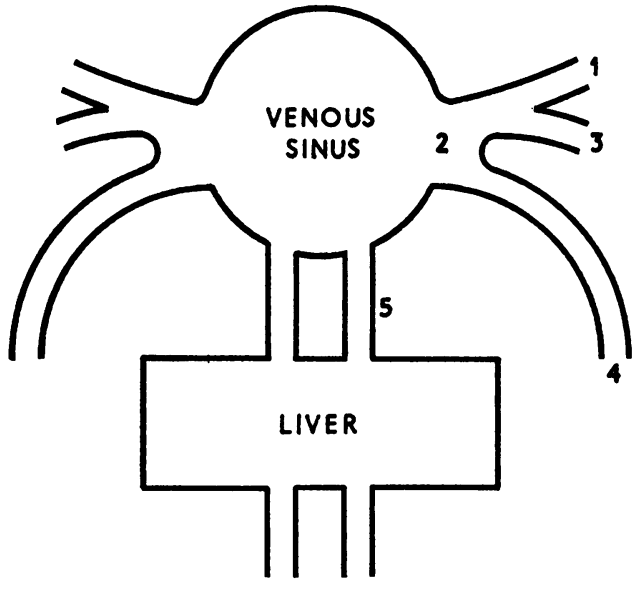

FIG. 4 Diagrammatic representation of the bilateral and symmetrical stage in early cardiac and systemic venous development. $I=$ anterior cardinal vein; $2=$ common cardinal vein; $3=$ posterior cardinal vein; $4=$ umbilical vein; $5=$ vitelline vein.

while the left-sided structures normally atrophy and disappear. The vitelline veins fuse to become a single channel, the hepato-cardiac channel, which connects with the inferior vena cava and thereby forms a single channel to the heart. Failure of disappearance, or reduction in size of the left sinus will permit not only persistent drainage of the anterior cardinal vein (the left superior vena cava) but will permit persistent drainage of the left vitelline vein which continues to drain into this channel. Thus hepatic venous blood from the left lobe of the liver will join the left superior caval and coronary venous blood to enter the right atrium so that no physiological abnormality exists.

Snellen and Bruins (1968) state that hepatic veins may occasionally open separately into the right atrium or coronary sinus but do not comment further. As far as we can ascertain, previous reports of persistence of the left superior vena cava draining into the coronary sinus and right atrium do not mention additional drainage of a left hepatic vein into this channel. We presume that an additional factor in our patient has permitted continued drainage of the left vitelline vein (the left hepatic vein) as well as persistence of the duct of Cuvier.

We would like to thank Dr. S. Disler, Medical Superintendent of Wentworth Hospital, for permission to publish; and the Ethical Drug Foundation for continued financial assistance.

\section{References}

Edwards, J. E., Carey, L. S., Neufeld, H. N., and Lester, R. G. (1965). Congenital Heart Disease. W. B. Saunders, Philadelphia.

Fleming, J. S., and Gibson, R. V. (1964). Absent right superior vena cava as an isolated anomaly. British fournal of Radiology, 37, 696.

Gasul, B. M., Arcilla, R. A., and Lev, M. (1966). Heart Disease in Children. Lippincott, Philadelphia.

Harrison, D. C., and Morrow, A. G. (1963). Electrocardiographic evidence of left-axis deviation in patients with defects of the atrial septum of the secundum type. New England fournal of Medicine, 269, 743.

Hastreiter, A. R., and Rodriguez-Coronel, A. (1968). Anomalous inferior vena cava with azygous continuation, high (sinus venosus) atrial septal defect and alterations of sinoatrial rhythm. American fournal of Cardiology, 21, 575.

Keith, J. D., Rowe, R. D., and Vlad, P. (1967). Heart Disease in Infancy and Childhood, and ed. Macmillan, New York.

Kjellberg, S. R., Mannheimer, E., Rudhe, U., and Jonsson, B. (1959). Diagnosis of Congenital Heart Disease, and ed. Year Book Publishers, Chicago.

Moss, A. J., and Adams, F. H. (1968). Heart Disease in Infants, Children and Adolescents. Williams and Wilkins, Baltimore.

Nadas, A. S. (1963). Pediatric Cardiology, and ed. W. B. Saunders, Philadelphia.

Snellen, H. A., and Bruins, C. (1968). Anomalies of venous return. In Paediatric Cardiology, p. 416. Ed. by $\mathrm{H}$. Watson. Lloyd-Luke, London.

Taussig, H. B. (1960). Congenital Malformations of the Heart, 2nd ed. The Commonwealth Fund, Harvard University Press, Cambridge, Massachusetts.

Watson, H. (1968). Paediatric Cardiology. Lloyd-Luke, London.

Wood, P. (1968). Diseases of the Heart and Circulation, 3rd ed. Eyre and Spottiswoode, London. 